\title{
COVID-19: Frente al desafío pedagógico de lo presencial a lo virtual
}

\section{COVID-19: Facing the pedagogical challenge of physical attendance to virtual}

\author{
Kleeder J. Bracho Pérez ${ }^{1}$ \\ https://orcid.org/0000-0003-3644-0561 \\ Universidad de Pamplona, Colombia \\ Marianggeline Ch. Bracho Durán ${ }^{2}$ \\ https://orcid.org/0000-0001-9192-4660 \\ Universidad del Zulia, Venezuela
}

Recibido: 05-04-2020

Aceptado: 01-08-2020

\section{Cita Recomendada}

Bracho, K.J. \& Bracho, M. Ch., (2020). COVID-19: Facing the pedagogical challenge of physical attendance to virtual. Hamut'ay, 7 (2), 9-17.

http://dx.doi.org/10.21503/hamu.v7i2.2127

\section{RESUMEN}

El COVID-19 es una enfermedad de carácter infeccioso y contagioso, considerada pandemia esencialmente por la velocidad en su propagación, causando en los seres humanos infecciones respiratorias severas, que pasan por ser un resfriado común hasta enfermedades más comprometidas como síndromes respiratorios inflexibles que pueden terminar siendo mortales, teniendo la particularidad de propagarse de persona a persona.

Ante esta situación, las autoridades sanitarias y gubernamentales colombianas han hecho énfasis en las medidas de confinamiento, que buscan en la población un distanciamiento social, aislamiento y cuarentena, medida que busca por todo los medios mitigar el riesgo de contagio, por lo que llevó a la educación a desarrollar el aprendizaje a través de la virtualidad, contando actualmente con docentes y directivos con conocimientos suficientes en contenidos, didáctica, organización, administración, material didáctico, entre otros.

En cuanto a los actores educativos están obligados a cambiar de actitud y disposición para afrontar el gran desafío pedagógico que los ocupa, pero es hasta ahora que con gran preocupación las instituciones educativas en general, se han hecho conscientes de lo imperioso que es contar con un personal altamente preparado, capacitado y calificado en consideración al uso adecuado de las Tecnologías de Información y Comunicación.

Palabras clave: virus, educación, pedagogía, tecnología, aprendizaje virtual.

1 Doctor en Ciencias de la Educación. Docente. Universidad de Pamplona. Colombia. E-mail: kleeder8@hotmail.com 2 Abogado Docente. Universidad del Zulia. Venezuela. E-mail: marianggeline@gmail.com 


\section{Abstract}

COVID-19 is an infectious and contagious disease, considered a pandemic essentially due to the speed of its spread, causing severe respiratory infections in humans, which happen to be a common cold to more compromised diseases such as inflexible respiratory syndromes that can end up being mortal, having the particularity of spreading from person to person.

In this situation, the Colombian health and government authorities have emphasized confinement measures, which seek social distancing, isolation and quarantine in the population, a measure that seeks by all means to mitigate the risk of contagion, which is why I have to education to develop virtual learning, currently having teachers and managers with sufficient knowledge in content, didactics, organization, administration, didactic material, among others.

As for educational actors, they are obliged to change their attitude and disposition to face the great pedagogical challenge that occupies them, but it is up to now that with great concern, educational institutions in general have become aware of how urgent it is to have a highly prepared, trained and qualified personnel in consideration of the proper use of Information and Communication Technologies.

Keywords: virus, education, pedagogy, technology, virtual learning.

\section{INTRODUCCIÓN}

Actualmente el mundo afronta una emergencia sanitaria provocada por el COVID-19, enfermedad de carácter infeccioso y contagioso, conocida también como enfermedad por coronavirus, originada por el virus (SARS-CoV-2.910), detectada por vez primera en la localidad de Wuhan en China. Organización Mundial de la Salud (OMS, 2019), en la actualidad esta enfermedad se expandió por todo el mundo, declarada por la organización antes mencionada como pandemia, reseńa que hace Sevillano, (2020), en el que afirma en palabras de su máximo representante el "Dr. Tedros Ghebreyesus, quien ha declarado el virus de coronavirus como pandemia ante el aumento de casos en todo el mundo".

Esta enfermedad, es considerada pandemia básicamente por la forma tan veloz de su propagación, causando en los seres humanos infecciones respiratorias severas, que pasan por ser un resfriado común hasta enfermedades más comprometidas como síndromes respiratorios inflexibles que pueden terminar siendo mortales, de igual modo, tiene la particularidad de propagarse de persona a persona, a través de las gotículas que proceden de la boca o la nariz saliendo expulsadas cuando la persona contagiada estornuda, exhala o tose.

Ante esta situación y las cifras alarmante de mortalidad en otros países, como Italia, España, Ecuador y EEUU, entre otros, ha despertado en las distintas autoridades sanitarias y gubernamentales de los países gran preocupación, en cuanto han sido duramente afectados en lo económico, lo social y lo cultural, entre otros, por lo que han tenido que hacer cambios rápidos, a fin de seguir aportando de forma inmediata medidas de protección para sus poblaciones.

Es de destacar, entre las medidas que recomiendan los organismos internacionales de salud, enfatizan en la higiene regular y completa de las manos, de las vías respiratorias, mantenerse informado y acatar las medidas de confinamiento, que buscan minimizar la propagación de la enfermedad.

Del mismo modo, las autoridades sanitarias y gubernamentales colombianas, han hecho énfasis en las medidas de confinamiento, que buscan en la población un distanciamiento social, aislamiento 
y cuarentena, medida que busca por todos los medios mitigar el riesgo de contagio, haciendo eco el Ministerio de salud y Protección Social (Resolución 385 de 2020. Art. 598), en el que establece que "toda persona debe velar por el mejoramiento, conservación y recuperación de su salud personal y la salud de los miembros de su hogar, evitando acciones y omisiones perjudiciales, cumpliendo las instrucciones técnicas y normas obligatorias que dicten las autoridades".

Asimismo, ante estas disposiciones reglamentarias se hizo efectiva la suspensión de todas las actividades de clases presenciales, para todas las instituciones de educación en los distintos niveles y modalidades. Ante la situación de confinamiento y suspensión de las actividades escolares, el presidente de la República de Colombia Iván Duque Márquez (2020), confirmó que los estudiantes "no tendrán más clases presenciales para salvaguardar la salud de todos" además señaló que "profesores y directivos deberán emprender la búsqueda de planes que les permitan a los jóvenes continuar con sus labores desde casa". En consecuencias, los docentes y directivos han tenido que voltear la mirada a buscar alternativas para que el calendario escolar no resulte severamente afectado por cuenta de la pandemia.

Aunado a lo anteriormente descrito, la colectividad y los actores educativos como alumnos, docentes y directivos, fueron sorprendidos ante la medida de confinamiento por la suspensión de las actividades de clase presenciales, el cual los ha conducido a enfrentar un gran desafío que no pueden relegarse, ante la exigencia de la virtualidad y la situación que se enfrentan, haciéndose participes de grandes retos.

Esta situación, les ha permitido adentrarse en la tecnología en las comunicaciones, con un avance en la hipercomunicación, la multimedialidad, equipos y software, redes entre otros, haciéndolos participes de una sociedad moderna y haciendo uso de nuevas alternativas de aprendizaje, según Lolas, Rodríguez y Valdebenito (2008, p.3), afirman que "la enseñanza virtual se basa en un nuevo modelo educativo, en que el aprendizaje no es una copia o reproducción pasiva de la información recibida, sino que demanda un proceso activo de interacción y relación de los nuevos contenidos con los que ya se poseían." Es decir, los actores educativos hacen incursión en el modelo educativo interactivo.

Ante esta nueva modalidad educativa, que inserta la virtualidad lleva a los estudiantes, docentes y directivos a realizar serios cuestionamientos en el que se aquejan entre ellos mismos, pudiéndose concebir una resistencia al cambio, según Faria (2005, p. 62), refiere que esta resistencia "puede ser causada por negación de la realidad como mecanismo de defensa. La tendencia a percibir solamente aquello que conviene". Lo que conlleva a impedir en gran medida dar el salto requerido, para afrontar el reto de una educación a distancia con criterios virtuales que se hace imperiosa ante la situación por la que vive el mundo.

Aunado a lo anterior, Bracho (2019), señala que la educación de las nuevas generaciones, deben fundamentarse en el desarrollo de las capacidades de invención en la solución de problemas, de la adaptación de procesos, y del análisis de situaciones, Ante este escenario, lleva a desarrollar todo esfuerzo humano intencional y ordenado, donde interactúen los actores educativos bajo condiciones de exigencias de flexibilidad en el proceso de educación a distancia, por lo que solo se quiere atender a intereses particulares, personales, colectivos y organizacional, según Vander (2000, p. 312), señala que esta actitud es "una predisposición adquirida y relativamente duradera a evaluar de determinado modo." Es decir, una tendencia subyacente a reconocer algo de manera propicia o desfavorable.

En tal sentido, hay muchos actores que no utilizan la tecnología de manera adecuada, se habla de un recurso que funciona a través de una computadora o aparatos móviles, pero siempre existe una excusa para no integrarse a una actividad educativa virtual, mostrando desinterés, Weinstein y Mayer (2002), expresan que más allá del aprendizaje de una faena o profesión, concierta en un sentido más vasto, obtener componentes que consientan hacer frente a situaciones nuevas y que ayude a realizar el trabajo. Los actores educativos, necesitan cambiar su actitud y disposición, para afrontar este nuevo desafío, se debe estar convencidos de la calidad, la efectividad, modalidad mediatizadas por la virtualidad que se realiza. 


\section{MÉTODO}

El actual articulo presenta un fundamento teórico, basado en una revisión exhaustiva de literatura, artículos científicos y de revisión, que posterior a su lectura conllevaron a realizar análisis e interpretación del desafío pedagógico que enfrentan la educación en relación a la pandemia del COVID -19, que afecta al mundo. Cabe destacar, que dicha revisión contó con información importante extraída de bases de datos como: Redalyc, SciElo, Dialnet, Elservier, PubMed, Google Books y Google Scholar, biblioteca virtual de CONCYTEC, repositorios virtuales nacionales e internacionales, entre otros, tomando en consideración los siguientes descriptores extraídos del tesauro de la UNESCO: i. Virus, ii. Educación, ii. Pedagogía, iv. Tecnología, v. Aprendizaje Virtual.

Asimismo, se llevó a cabo el análisis del campo semántico a partir de la búsqueda de los tesauros, ejemplo de campo semántico sería el de términos relacionados con los descriptores descritos en la tabla 1:

\section{Tabla 1}

Campo Semántico

\begin{tabular}{|c|c|c|}
\hline Palabra & Sema Compartido & Semas Distinguidores \\
\hline Virus & $\begin{array}{l}\text { Contaminante viro- } \\
\text { logía, bacteria }\end{array}$ & $\begin{array}{l}\text { Enfermedad Trasmi- } \\
\text { sible }\end{array}$ \\
\hline Educación & $\begin{array}{l}\text { Aprendizaje, do- } \\
\text { cencia }\end{array}$ & $\begin{array}{l}\text { Desarrollo de habilida- } \\
\text { des y actitudes }\end{array}$ \\
\hline Pedagogía & $\begin{array}{l}\text { Metodología, } \\
\text { practica. }\end{array}$ & Método de Enseñanza \\
\hline Tecnología & $\begin{array}{l}\text { Recursos, equi- } \\
\text { pamiento, conoci- } \\
\text { miento }\end{array}$ & $\begin{array}{l}\text { Instrumentos, métodos } \\
\text { y técnicas }\end{array}$ \\
\hline $\begin{array}{l}\text { Aprendizaje } \\
\text { Virtual }\end{array}$ & $\begin{array}{l}\text { Enseñanza asistida, } \\
\text { internet. }\end{array}$ & $\begin{array}{l}\text { Aprendizaje a distancia } \\
\text { vía el Internet }\end{array}$ \\
\hline
\end{tabular}

Fuente: Elaboración propia (2020).

La revisión de la literatura y posterior análisis se realizó debido a la mayor fuerza de contagio de la pandemia del COVID-19 y en el cual se expandió hacia América Latina, afectando a sus habitantes y a todos los sectores productivos de los países, incluyendo la educación, sector el cual ha tenido que reinventarse pedagógicamente y acudir a la tecnología con mayor énfasis para poder mantenerse y cumplir con sus fines y propósitos. Por otra parte, el actual artículo se caracteriza por ser una revisión documental, según Hurtado (2008), afirma que una revisión documental es una técnica donde se recolecta información escrita sobre un determinado tema. En este sentido en vista que se partió de una exploración teórica sobre el tema en cuestión está organizado desde una introducción, desafío pedagógico, actores educativos y el aprendizaje virtual, por lo que no ha requerido hacer una descripción de cada uno de los artículos, ya que todos fueron citados, analizados y están referenciados.

\section{Desafío Pedagógico}

Actualmente, ante la situación de confinamiento y suspensión de las actividades escolares, los actores educativos han tenido que enfrentar un gran reto, un desafío, más allá de las actividades cotidianas estructurales, organizativas que se venían desarrollando, afín de darle otro sentido a los procesos pedagógicos sin desvirtuar la concepción objetiva de la educación. Piaget (1983, p. 9), señala que "el objeto principal de la educación es crear hombres que sean competentes de hacer nuevas cosas, no repetir meramente lo que han habituado las demás generaciones, hombres que sean creativos, inventivos y descubridores". Bajo esta afirmación que hace el autor anteriormente citado, los actores educativos están llamados a afrontar la crisis que vive el mundo hoy, haciendo lo que mejor saben hacer dentro del ámbito educativo, pero de forma diferente.

Esta situación de crisis, que tomó por sorpresa a todos los campos sociales y en especial a la educación, donde la mayoría de sus actores han tenido que abrirse a explorar nuevas posibilidades y romper esquemas, que por años han transgredido el uso de las Tecnologías de Información y Comunicación, sin concebir su uso para orientar el aprendizaje, Ortega (2004), hace severos señalamientos sobre el auge de las tecnologías, el cual ha generado esperanzas a la humanidad, colocado instrumentos de comunicación que favorecen la extensión de la cultura, la educación, la democracia y el pluralismo.

Aunado a lo anteriormente descrito, para la mayo- 
ría de los actores educativos ante el obligado uso de las tecnologías de información y comunicación, ha incidido en la mente de éstos, considerando que deben enfrentarse a un gran cambio, donde los alumnos, docentes y directivos escasamente cuentan con la capacitación adecuada, para el uso de las tecnologías y como orientar contenidos educativos de forma virtual, según Cabero (2006), afirma que "las ventajas de incorporar las TIC en la educación son cada vez más diversas", beneficiando y fortaleciendo las áreas para el aprendizaje cooperativo.

La gran mayoría de éstos actores conciben de forma empírica el uso de la tecnología, un uso limitado en el que solo se destacan las redes sociales y en su defecto el correo electrónico, dejando de lado las competencias que pudiera adquirir en el desarrollo de actividades educativas online, a través del uso de herramientas que fortalezcan la praxis educativa, por lo que se requiere de formación o capacitación de ellas, Barberá (2003, p.32), señala "que uno de los factores más significativos sea la formación y el perfeccionamiento que el profesorado tiene para su integración en los diferentes contextos de aprendizaje", es decir, que la formación y capacitación debe considerarse elementos decisivos en el proceso educativo.

El aprendizaje a través de la virtualidad no había sido necesario como hoy día, actualmente contamos con docentes y directivos con conocimiento suficientes en contenidos, didáctica, organización, administración, material didáctico, entre otros, pero son muchos los que no cuentan con competencias para desarrollar actividades de clase en la modalidad virtual, no se convencen de la efectividad de la modalidad mediatizada por la virtualidad, conllevándolos a generar estrés, Papalia, Wendkos y Duskin (2005), señalan que el estrés refiere a una emoción negativa, generando ansiedad o estado de ánimo depresivo, que se asocia a una mala salud mental y física. Es decir, se logra obtener poca motivación, menos productivo, se tiene menos de lo que hace, por lo que conlleva a tener pocas perspectivas de éxito.

Ante la situación presentada, los actores educativos están obligados a cambiar de actitud y disposición para afrontar el gran desafío pedagógico que los ocupa, pero es hasta ahora que con gran preocupa- ción las instituciones educativas en general, se han hecho conscientes de lo imperioso que es contar con un personal altamente preparado, capacitado y calificado en consideración al uso adecuado de las tecnologías de información y comunicación, según Salinas, (2004,p.6), señala "que aquellas universidades que no contemplan cambios radicales, en relación a los medios didácticos y a los sistemas de distribución de la enseñanza, pueden quedar fuera de la corriente innovadora”, en tal sentido, es imperante propiciar un cambio radical, valorar el uso adecuado y consciente de la tecnología.

\section{Aprestamiento Tecnológico}

Al considerar el proceso de aprestamiento de la tecnología en la educación, es de suma importancia reflexionar sobre la actitud que deben asumir los actores educativos frente al cambio, que lleva consigo una preparación y reestructuración continua de los procesos pedagógicos que desarrollan, manteniendo activa la comunicación como un hecho principal de la formación, es de destacar, que no es fácil cambiar de paradigma para la aplicación de la educación en los procesos de formación y aprendizaje a través de la tecnología.

Este aprestamiento tecnológico, Álvarez (2007, p.14), conlleva un conjunto de actividades organizadas, que promueven en dichos actores el desarrollo de habilidades, destrezas y la adquisición de hábitos y actitudes positivas hacia la utilización de las Tecnologías de Información y Comunicación (TIC) para alcanzar el nivel de éxito en el aprendizaje. De esta forma, el aprestamiento tecnológico, busca la preparación psicomotriz que suscite la sensibilización y calificación de algunas competencias, a ser desarrolladas en las distintas fases escolares cuando es empleada la educación a través de la tecnología.

El aprestamiento de la tecnología en la educación no la hace de menor calidad, ni de baja intensidad en los contenidos que la educación presencial, por el contrario, se concibe como un ámbito de gran importancia para el empoderamiento de otros conocimientos y la apropiación de nuevas capacidades y habilidades, como lo refiere Mara- 
botto y Grau (2002, p.19), la comprensión global del recurso, sensibilización y familiarización a fin de convertirse en usuario inteligente, capaz de cambiar parámetros en los procesos de formación y evaluación creando un rol docente diferente al tradicional, en una educación despresencializada y en correspondencia con la situación actual que se vive en Latinoamérica y el mundo con respecto a la pandemia del COVID-19.

En tal sentido, el dominio adecuado de la tecnología por parte de docentes y estudiantes, hace que los contenidos, aplicaciones o herramientas utilizadas en este sistema educativo virtual aporte al mejoramiento eficiente del proceso de aprendizaje y el desempeño del docente en sus actividades diarias, pero entran en un conflicto con el acceso a la conectividad como uno de los puntos clave para el desarrollo normal y eficiente de las actividades virtuales. el Banco Mundial (2019), advierte que "para evitar este escenario, los países esperan intensificar sus esfuerzos hacia el acceso universal de la banda ancha, $y$ dotar a las personas de las habilidades y recursos que necesitan para participar plenamente en la economía digital", por lo que se hace necesario una revisión de las políticas de estado en materia de Tecnologías de Información y Comunicación para ir ajustándose a estos requerimientos.

Es de hacer notar, que el uso de las Tecnologías de Información Comunicación en el sector educativo, debe superar la visión limitadora del computador, y abrir paso a la integración de las distintas formas de comunicación que incluyen las redes sociales y que pueden ser útiles a la gestión de conocimiento en el proceso permanente de aprendizaje. Gutiérrez (2017), alude a la relación de las instituciones educativas con las redes sociales y señala que la educación no puede quedarse fuera de las redes sociales y que debe ser un aspecto cultural muy relevante, es decir educar de acuerdo a las necesidades del entorno y a lo que el avance del mundo ofrece.

Este proceso de aprestamiento de la tecnología en la educación, debe considerar todas las necesidades de los contextos, a fin de apropiarse de habilidades y convertirse en una herramienta para crear grupos de aprendizajes dinámicos y activos, para potenciar la autonomía en el aprendizaje para el cambio de las prácticas convencionales de construir y pro- ducir conocimiento. Tellería (2009), señala que los continuos avances de la tecnología dan origen a diferentes procesos de comunicación que estimulan interacciones diversas que impulsan al sistema educativo. Lo que constituye una alternativa suprema para la formación y redimensión de los procesos de comunicación y del aprendizaje.

\section{Actores Educativos y El Aprendizaje VirTUal}

En la actualidad son muchas las persona que utilizan la tecnología, unos de manera adecuada y otros que desvirtúan funcionabilidad de este recurso, las computadoras y los móviles, se han convertido en unos instrumentos que captan la atención de los seres humanos. Convirtiéndose en un constante desafío cuando se trata de asumir el compromiso y la responsabilidad de un proceso de aprendizaje mediado a través de la virtualidad, en este caso atendiendo a la necesidad imperante por la que atraviesa el mundo ante la pandemia del COVID-19.

Ante esta situación los actores educativos se ven obligados a explorar posibilidades nuevas, que permiten adentrarse a una educación asistida en red, Medina (2013), enfrentarse a una experiencia que los conduce a enriquecerse en la forma de ver al mundo, la cultura y la sociedad, llevándolos a reflexionar sobre la responsabilidad que se tiene, ante la sociedad en su proceso formativo; indistintamente ante la sociedad y en el rol que le concierna.

$\mathrm{Al}$ hacer referencia a los actores llámense estos estudiantes, docentes y directivos de instituciones de educación, se han cuestionado su preparación en la modalidad virtual, haciéndose conscientes de la brecha que existe entre el uso adecuado de las tecnologías de información y comunicación con la realidad existente en cada uno de ellos. García (2002), señala que haciendo uso de las tecnologías es viable llevar los procesos de aprendizaje críticos e innovadores o reproducir las estructuras de comunicación de la educación tradicional.

Aunado a lo expuesto anteriormente, se deben aprovechar las posibilidades que ofrece el medio para adecuar el aprendizaje y manejo adecuado de 
las herramientas tecnológicas, para fundar cooperativamente los significados y obtener así un conocimiento profundo, responsabilizándose de su propio aprendizaje siendo el conocimiento un provecho subjetivo e individual de la innovación de la información, en consecuencias Pineda, Casanova \& Bracho, (2020), afirman que las tecnologías son esenciales para favorecer el desarrollo de las dimensiones y competencias en todo proceso de cambio e innovación, los cuales solventan los requerimientos actuales de una sociedad globalizada.

Por otra parte, el docente es parte fundamental, por lo que éste debería contar con las competencias sobre herramientas tecnológicas, según Harasim (2000), el aprendizaje debe estar centrado en el educando y demanda un papel disímil del profesor. Asimismo, (Paulsen, 1992 en Cabero, 2001), señala que el rol del docente debe basarse esencialmente en la dinamización del curso y en tomar funciones de control y organización de las actividades, que motiven y que proporcione experiencias para el auto-aprendizaje y la cimentación del conocimiento bajo la interacción asincrónica entre alumno, docente y los contenidos. Torres, (2020), señala que la incorporación de las tecnologías y las herramientas digitales han permitido una gran transformación en la educación porque mejora la calidad educativa y la forma de ver el mundo desde una realidad construida por la sociedad desde una manera individualizada.

En el caso de los estudiantes, su rol debe ser más activo, el cual es vital para enriquecer su propio proceso de aprendizaje, puesto que deja de ser un receptor de información a convertirse en un investigador y administrador de la información que consigue para aprehender los contenidos abordados en el curso, generando de esta manera un gran aprestamiento informático, Stojanovic (2001), afirma que ese aprestamiento informático es una característica dentro de la actividad virtual, empleando de manera eficiente todas las funcionalidades ofrecidas para producir acciones formativas de modo flexible e independiente del tiempo y espacio. Otra de las responsabilidades o rol que asume el estudiante es administrar su propio tiempo dedicado al aprendizaje, enmarcado en el horario que deberá leer, estudiar e investigar para cumplir con sus asignaciones y tareas, por lo que ya no es el docente quien establece los límites de información sino es él mismo estudiante.

Por último, el personal rector que dirigen las instituciones educativas han indicado instrucciones precisas sobre la continuación de los planes de estudio a través de campus virtuales, medios de comunicación u otros ambientes digitales, así como en algunos casos la reprogramación de los calendarios académicos, ante estos lineamientos asumen con mucha preocupación que el personal a su cargo poseen escasa competencias en el manejo de las herramientas tecnológicas y hasta ahora que se trabaja la idea de capacitar a los docentes, según Quintero (2018), señala que "había que tener talento humano preparado para que no fuera un fracaso la educación virtual. Necesitamos tiempo para cualificar a nuestros docentes"

En tal sentido, ante la situación que enfrenta el personal rector de las instituciones educativas, han tenido que actuar ante un plan de contingencia con el asesoramiento sobre las herramientas tecnológicas virtuales a docentes y estudiantes. Por su parte, Roca (2002, p.22) manifiesta que "el desempeńo profesional está asociado al cumplimiento de las obligaciones, funciones y papeles de la profesión ejercida por un individuo, demostrando rapidez, exactitud, precisión y cuidado en el proceso de ejecución”. De esta forma, el desempeño de las instituciones, grupo o persona está determinado por una composición sistémica de lo que correspondió concebir en el pasado, lograr en el presente y podría concebirse en el futuro; entendiendo el logro como una función integrada entre objetivos, resultados, competencias y comportamientos.

\section{Conclusiones}

Una vez hecho el análisis e interpretación del desafío pedagógico que enfrentan la educación en relación a la pandemia del COVID-19, se hace la reflexión y concluyendo respecto a la problemática. Es imperioso que el sector de la educación a través de las tecnologías de información y comunicación, se eleven las recomendaciones necesarias para mitigar el impacto que ocasiona la epidemia que hoy afecta al mundo y sensibilizar los riesgos de la en- 
fermedad, en los distintos sectores de la sociedad, siendo la educación un canal propicio para llegar a más lugares y despertar la conciencia ciudadana desde distintos hogares. Asimismo, los actores que forman parte de los procesos educativos están llamados a reinventarse pedagógicamente, haciendo lo que mejor saben hacer dentro de su ámbito educativo, pero de forma diferente a través de estrategias que involucren el uso de las tecnologías y actividades virtuales impulsando de esta manera la práctica del conocimiento, el trabajo autónomo y asistido.

Por otra parte, contextualizar la importancia sobre el empleo de las tecnologías en las diferentes actividades educativas que desarrollan docentes y estudiantes, bajo entornos virtuales de aprendizaje como apoyo a los procesos de formación en los distintos niveles y modalidades del sistema educativo.

Es de considerar también que los cambios pedagógicos, el aprestamiento tecnológico y comunicacionales se encuentran en una constante evolución, por lo que se requiere de inversiones personales e institucionales para la capacitación de los actores educativos respecto al uso eficiente de las tecnologías, manejo de herramientas y adecuación de las mismas para orientar los procesos educativos de forma virtual.

Es de hacer notar, que los actores educativos escasamente hacen uso de aplicaciones de materiales tecno digitales en sus actividades presenciales, por lo que para el momento no se fomentó la educación virtual, ante la situación de pandemia dichos actores se han visto de manera forzosa e improvisada a asumir el uso de herramientas virtuales para garantizar el acceso a la educación.

\section{REFERENCIAS BIBLIOGRÁFICAS}

Álvarez, A. (2007). Hacia un enfoque cognitivo del diseño industrial: Una aproximación psicogenética del desarrollo cognitivo mediante la interacción con objetos tecnológicos. $2^{\circ}$ Encuentro Latinoamericano de Diseño 2007, Facultad de Diseño y Comunicación, Universidad de Palermo, Buenos Aires, Argentina.

Banco Mundial (2019), Tecnologías de información y las Comunicaciones. Recuperado de: https://www.bancomundial. org/es/topic/digitaldevelopment/overview
Barberá, E. (2003). Estado y tendencias de la evaluación en educación superior. Revista de Docencia Universitaria, Vol.3(2), 94-99.

Bracho, K. (2019). La Cultura de la Investigación y la Producción Científica, Un acercamiento a la realidad de las universidades privadas. Editorial Académica Española. España.

Cabero, J. (2006). Bases pedagógicas del e-learning. Catalunya. Revista de Universidad y Sociedad del Conocimiento (RUSC), Vol. 3 (1). 01-10. https://doi.org/10.7238/rusc.v3i1.265

Cubero, R. (2001). Perspectivas constructivistas. La intersección entre el significado, la interacción y el discurso. Barcelona.

Duque, I. (2020). Suspensión de clases en colegios y universidades por el COVID-19. Periódico La Opinión. (15 de marzo de 2020). Recuperado de https://www.laopinion. com.co/colombia/duque-anuncia-suspension-de-clases-encolegios-y-universidades-por-el-covid-19-193568\#OP

Faria, F. (2005), Desarrollo Organizacional. Enfoque Integral. México, Limusa.

García, A. (2002). El debate telemático como experiencia de aprendizaje en el aula universitaria, en EDUTEC'99. Nuevas Tecnologías en la Formación Flexible y a distancia. Sevilla, SAV.

Gutiérrez, F. (2017). Educación para la sostenibilidad de España. Reflexiones y Propuestas. Recuperado de: http:// eaxxi.blogspot.com/2017/10/benayas-marcenalba-y-gutierrez-bastida.html.

Harasin, D. (2000). "Network Learning: A Paradigm for the twenty-first Century".Learning Netwoks. The MIT Press: Cambridge, M A., 271-278.

Hurtado J. (2008). El Proyecto de Investigación. Ed Quirón. Caracas - Venezuela.

Lolas F., Rodríguez, E. y Valdebenito, C. (2008). Enseñanza virtual de la bioética. Desafíos. Acta Bioethica, 14(1), P. 47-53. https://doi.org/10.4067/S1726-569X2008000100006

Marabotto, M., y Grau, J. (2002). Hacia la informatización del aprendizaje. FUNDEC, Buenos Aires - Argentina.

Medina, M. (2013). E-Learning y Desempeño Laboral del Supervisor en Educación Primaria. (trabajo de grado). Universidad Rafel Urdaneta. Maracaibo - Venezuela.

Ministerio de Salud y Protección Social (Resolución 385 de 12 marzo 2020). Se declara la emergencia sanitaria por causa del coronavirus COVID-19 y se adoptan medidas para hacer frente al virus.

Ministerio de salud y Protección Social (Resolución 380 de 10 marzo 2020). Medidas preventivas sanitarias en el país, por causas del coronavirus- COVID2019.

Ortega, P. (2004). La educación moral como pedagogía de la alteridad, Revista Española de Pedagogía, 227, 5-30.

Papalia, D, Wendkos, S y Duskin, R. (2005). Desarrollo Humano. (9a. ed.) México: McGrawHill. 
Piaget, J. (1983). El Criterio Moral en el Niño. Barcelona: Editorial Fontanella

Pineda, W., Casanova, M. \& Bracho, K., (2020). Objeto virtual de aprendizaje en la Cátedra de Paz para estudiantes de educación básica primaria. Hamut'ay 7(1), 9-18.

https://doi.org/10.21503/hamu.v7i1.1894

Quintero, I. (2018), Docentes deben capacitarse para la educación virtual. Periódico La Crónica (febrero 05/2018).

Recuperado de https:/www.cronicadelquindio.com/ noticia-completa-titulo-docentes-deben-capacitarse-para-la-educacion-virtual-nota-117778

Roca, A. (2002) Modelo de mejoramiento del desempeño pedagógico profesional de los docentes que laboran en la ETP. Tesis de Doctorado. ISP Holguín.

Salinas, J. (2004). Innovación docente y uso de las TIC en la enseñanza universitaria Revista de Universidad y Sociedad del Conocimiento (RUSC), Vol. 1 (1).

Sevillano, E. (2020). La OMS declara el brote de coronavirus pandemia global. Periódico El País. (11 de marzo de 2020). Recuperado de https://elpais.com/sociedad/2020-03-11/laoms-declara-el-brote-de-coronavirus-pandemia-global.html

Stojanovic, L. (2001). Mejoramiento del aprendizaje a través de las nuevas tecnologías de información en la educación a distancia. Revista Iberoamericana de Educación a Distancia. Volumen 4. Diciembre.

Tellería, M. (2009). Las nuevas tecnologías: posibilidades para el aprendizaje y la investigación. Teoría y Didáctica de las Ciencias Sociales, 15, (479-502).

Torres, J. (2020), Sotfware Educativo para el Fortalecimiento del Aprendizaje de la Lengua Castellana en Estudiantes de Educación Básica Primaria. (trabajo de grado). Universidad de Pamplona. Cúcuta - Colombia.

Vander, Z. (2000), Manual de psicología social - Editorial Paidós - Buenos Aires.

Weinstein C. y Mayer R. (2002). La enseñanza de estrategias de aprendizaje. En M. C, Wittrock (ed.): Manual de investigación sobre la enseñanza, Nueva York, MacMillan. 